\title{
Associação entre a sintomatologia dolorosa e a disfunção temporomandibular
}

\author{
Association between painful symptomatology and temporomandibular dysfunction
}

Asociación entre la sintomatología dolorosa y la disfunción temporomandibular

Wyllka Cyntya Galvão da SILVA ${ }^{1}$

Karolinne Domingos de MEDEIROS ${ }^{1}$

Eloisa Cesário FERNANDES ${ }^{1}$

Sandja Gabriela Gomes de OLIVEIRA ${ }^{1}$

Caio Rodrigues MAIA ${ }^{1}$

Laio da Costa DUTRA ${ }^{2}$

Eduardo José Guerra SEABRA ${ }^{3}$

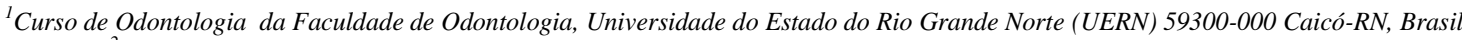

${ }^{2}$ Doutorando em Odontologia pela Universidade Estadual da Paraíba (UEPB) 58429-000 Campina Grande-PB, Brasil

${ }^{3}$ Professor Adjunto IV, Universidade do Estado do Rio Grande Norte (UERN) 59300-000 Caicó-RN, Brasil

\section{Resumo}

Introdução: A Disfunção Temporomandibular (DTM) é um quadro patológico que afeta o sistema estomatognático e, frequentemente, acompanhada de dor. Objetivos: Aferir o nível de dor dos pacientes portadores de DTM e a prevalência de sintomatologia articular e muscular. Percebeu-se que a relação entre a origem da DTM e a sintomatologia dolorosa é pouco relatada na literatura. Materiais e Métodos: Trata-se de um estudo descritivo, observacional de corte transversal, desenvolvido com 30 pacientes com DTM, diagnosticado pelo Research Diagnostic Criteria for Temporomandibular Disorders (RDC/TMD). A amostra foi determinada pelas estimativas de atendimento. A associação entre a sintomatologia e a origem da DTM foram verificadas através do teste qui-quadrado, com intervalos de confiança (95\%). Foi usada uma ficha clínica para a coleta de dados com idade, gênero e nível da dor, que foi aferido através da Escala Visual Analógica de dor. Resultados: Constatou-se que 26 pacientes eram do sexo feminino e 4 do sexo masculino. Quinze apresentaram idade inferior a 36,5 anos, e os outros uma idade superior a esta. Quanto a origem da DTM, 19 tinham desordem articular e 11 muscular. A categoria moderada foi o nível mais prevalente, seguida do intenso. Determinou-se que não houve associação estatisticamente significante entre as variáveis deste estudo com a DTM. Conclusão: Constatou-se que, os fatores etiológicos analisados isoladamente não influenciam de forma única no desenvolvimento da DTM, mas poderão atuar em conjunto com outros fatores, já que a sua causa é multifatorial.

Descritores: Dimensão Vertical; Boca Edêntula; Dor Facial; Síndrome da Disfunção da Articulação Temporomandibular.

\section{Abstract}

Introduction: The Temporomandibular Disorders (TDM) is a disease that affects stomatognathic system and, often, is associated with pain. Objective: To assess the standard of pain from patients with TMD and the prevalence of articular and muscular painful symptoms. The association between TMD and painful symptoms were scarcely related in literature. Materials and Methods: Cross-seccional observational study developed with 30 patients with TMD, diagnosed by Research Diagnostic Criteria for Temporomandibular Disorders (RDC/TMD). Attendance estimative determined the sample. The connection between origin and symptoms of TMD was verified by chi-square calculator with confidence level (95\%). To collect the data like genre, age and pain level, a clinical form and Visual Analogical Scale of Pain were used. Results: Was found that 26 patients were women and 4 were men. Fifteen had less than 36,5 years and the others had more than this age. About the origin of TMD, 19 had articular disorder and 11 muscular. Medium level was more prevalent, followed by intense. There wasn't significant association between the variables of this study and TMD. Conclusion: According this study, etiological factors isolated analyzed does not influence in a unique way of TMD development, although may act together with other factors because multifactorial origin of TMD.

Descriptors: Vertical Dimension; Mouth, Edentulous; Facial Pain; Temporomandibular Joint Dysfunction Syndrome.

\section{Resumen}

Introducción: La disfunción temporomandibular (DTM) es un cuadro patológico que afecta al sistema estomatognático ya menudo acompañado de dolor. Objetivos: Aferir el nivel de dolor de los pacientes con DTM y la prevalencia de sintomatología articular y muscular. Se percibió que la relación entre el origen de la DTM y la sintomatología dolorosa es poco relatada en la literatura. Materiales y Métodos: Es uno estudio, observacional, descritivo, transversal, involucrando 30 pacientes con DTM, diagnosticados por la Research Diagnostic Criteria for Temporomandibular Disorders (RDC/TMD). La muestra fue determinada por las estimaciones de tratamiento. La asociación entre la sintomatología y origen de la DTM se verificó a través del test chi-cuadrado, con intervalos de confianza (95\%). Se utilizó una ficha clínica para la recolección de datos con edad, género y nivel del dolor, que fue aferido a través de la Escala Visual Analógica de dolor. Resultados: Se constató que 26 pacientes eran del sexo femenino y 4 del sexo masculino. Quince presentaron una edad inferior a 36,5 años, y los otros una edad superior a ésta. En cuanto al origen de la DTM, 19 tenían desorden articular y 11 muscular. La categoría moderada fue el nivel más prevalentes, seguido del intenso. Se determinó que no hubo asociación estadísticamente significativa entre las variables de este estudio con la DTM. Conclusión: Foe contastado que, los factores etiológicos analizados aisladamente no influencian de forma única en el desarrollo de la DTM, pero podrán actuar en conjunto con otros factores, ya que su causa es multifactorial.

Descriptores: Dimensión Vertical; Boca Edéntula; Dolor Facial; Síndrome de la Disfunción de Articulación Temporomandibular.

\section{INTRODUÇÃO}

A Disfunção Temporomandibular (DTM) é uma condição ou um conjunto de alterações clínicas que levam ao mau funcionamento do sistema estomatognático, por meio de alterações e danos que ocorrem nos músculos mastigatórios, músculos posturais da cabeça e pescoço, componentes ósseos, cartilaginosos e nervosos que compõem a Articulação Temporomandibular (ATM). Assim, o prejuízo de um ou mais desses componentes pode gerar impacto sobre todo o sistema estomatognático, o que leva ao estado de disfunção.

A DTM é a principal causa de dor orofacial de origem não odontogênica, sendo assim de origem músculo-esquelética ${ }^{1}$. Boa parte da população mundial possui algum tipo de disfunção desse componente, apresentando algum ruído ou outro sinal, como a própria dor, que pode ser na face ou na articulação temporomandibular ${ }^{2}$.

Embora a DTM seja investigada por várias áreas da saúde, no que diz respeito a sua etiologia, ainda não é relatado fatores completamente conclusivos e claros sobre sua origem, devido à sua multifatorialidade, existindo assim, dificuldades para limitação das causas da disfunção 
temporomandibular, porém, alguns fatores influenciam a sua presença, como, trauma articular, discrepâncias oclusais, hipermobilidade articular, problemas esqueléticos, hábitos para funcionais, fatores psicossociais e de comportamento ${ }^{3}$.

Com relação a prevalência de sinais de DTM (movimentos anormais da mandíbula, ruídos articulares e sensibilidade à palpação) na população em geral, varia em até $75 \%$. Aproximadamente $33 \%$ da população tem pelo menos um sintoma (dor facial, dor nas articulações) ${ }^{4}$. Estudos de Jorge et al. relatam que essa patologia é mais prevalente em indivíduos jovens e, principalmente, em mulheres de meia idade. $O$ que vai de encontro com outros estudos demonstraram uma alta prevalência de DTM em pacientes idosos, mesmo que usuários de próteses totais duplas. Como este grupo de indivíduos pode ser o mais afetado por variações clinicamente significantes na DVO, é possível que estejam associados com o desenvolvimento de DTM. Por exemplo, em indivíduos edêntulos reabilitados com próteses totais removíveis, a falta de equilíbrio neuromuscular durante a cinética mandibular e/ou a presença de relações oclusais instáveis pode influenciar em maior índice de acometimento de DTM, o que pode provocar dor, já que a contração dos músculos da articulação temporomandibular está com os padrões alterados por conta da prótese mal adaptada ${ }^{5}$.

O sintoma mais frequentemente relatado pelos pacientes portadores de DTM é a dor, que pode ocorrer quando se palpam os músculos mastigatórios, a АTM ou a região pré-auricular e geralmente é agravada pela mastigação ou por outra função da mandíbula, como movimentos de abertura e fechamento, e lateralidade e protrusão ${ }^{6}$.

Portanto, o objetivo deste estudo foi avaliar a associação da sintomatologia dolorosa e a presença da disfunção temporomandibular.

\section{MATERIAL E MÉTODO}

\section{- Caracterização da pesquisa}

Trata-se de estudo descritivo, observacional de corte transversal com pacientes portadores de DTM atendidos na Clínica de Odontologia da Universidade do Estado do Rio Grande do Norte (UERN). Este estudo visa verificar a associação entre a sintomatologia dolorosa com a presença DTM. A pesquisa foi submetida e aprovada por um Comitê de Ética em Pesquisa, através do parecer $\mathrm{n}^{\circ} 668.250$ CEP/UERN 2014.

\section{- População e amostra}

O serviço de atendimento clínico do Curso de Odontologia da UERN fornece uma assistência odontológica a população da Cidade de Caicó-RN e para a região do Seridó norte-rio-grandense. Trata-se de um serviço de referência em que o paciente recebe assistência de acordo com a sua necessidade, desde o nível primário, secundário e terciário. $\mathrm{O}$ presente estudo foi desenvolvido com 30 (trinta) pacientes com DTM, diagnosticado pelos RDC/TMD. Como critérios de inclusão para esta pesquisa, citamos os seguintes aspectos: pacientes adultos portadores de DTM ou que desenvolveram ao longo do tratamento odontológico realizado na universidade; pacientes de ambos os gêneros; pacientes com idade igual ou superior a 18 anos. Qualquer situação encontrada durante a pesquisa que não se enquadrasse nos critérios supracitados excluiu totalmente o paciente da pesquisa.

\section{- Instrumentos para coleta dos dados}

Para a realização desta pesquisa, foi utilizada uma ficha clínica, em que inicialmente foram coletados os dados preliminares como idade e gênero. A ficha era formada por três quesitos, em que se coletaram as informações referentes à presença ou ausência de sintomatologia através da Escala Visual Analógica de Dor (EVA). Esta é composta por uma numeração que expressa o nível de dor que o paciente está sentindo, possuindo uma numeração que varia de 0 a 10 , indicado sem dor no número 0 e máximo de dor no número $10 .^{7}$ Após o estabelecimento do nível de dor do sujeito da pesquisa, foi assinalada na ficha clínica a origem da DTM, sendo de origem articular ou muscular, diagnosticado pelo RDC/DTM. O segundo quesito avaliou a condição dentária do indivíduo, sendo: totalmente dentado; parcialmente desdentado com contenção posterior; parcialmente desdentado sem contenção posterior e totalmente desdentado, com ou sem prótese total. O terceiro quesito verificou se o paciente possuía uma dimensão vertical estabelecida, diminuída ou aumentada.

\section{- Procedimentos para a coleta dos dados}

A avaliação clínica foi realizada somente através do pesquisador principal. Foi questionado ao paciente a sua idade e assinalado na ficha clínica o gênero do paciente. Quando a dor estava presente, o nível da sintomatologia foi aferido através da EVA. Antes que o paciente informar o seu grau de dor, o pesquisador esclareceu os níveis de dor dos quais a escala apresenta, para que a resposta fornecida fosse confiável. Quando a dor for moderada, esta se classifica como o número 5. E os níveis foram informados ao paciente, em que de 1 a 2 , a dor é denominada leve, de 3 a 7 , a dor é chamada de moderada, e de 8 a 10 a dor é intensa. Ao informar o indivíduo sobre a EVA, ele falou de forma espontânea em que nível de dor se encontrava naquele momento da avaliação. Anteriormente diagnosticado à pesquisa, através dos RDC/DTM, a origem da DTM foi indicado na ficha clínica.

- Análise estatística

Os dados foram digitados e armazenados concomitantemente à coleta dos mesmos, em uma planilha eletrônica do Microsoft Excel 2013, sendo uma por paciente. Após a conclusão do trabalho de transcrição, se realizou a tabulação e organização dos 
dados em tabelas contendo a frequência simples e absoluta dos mesmos para análise descritiva. $\mathrm{O}$ banco de dados da pesquisa foi construído na plataforma do software SPSS ${ }^{\circ}$ (Statistical Package for Social Sciences) versão 22.0. Realizou-se a análise descritiva de todos os dados relativos às variáveis, como também associação entre a sintomatologia dolorosa com DTM, que foram verificadas através do teste estatístico qui-quadrado. Para verificar a magnitude dessas associações, utilizaram-se razões de prevalência e seus respectivos intervalos de confiança $(95 \%)$.

\section{RESULTADOS}

O nível de dor apresentado pelos pacientes na abordagem inicial foi avaliado através dos valores aferidos pela EVA. Objetivando uma melhor organização, os dados coletados foram quantificados, método utilizado em diversos trabalhos na literatura. Dessa forma, agruparam-se os níveis de dor em três categorias: 0 a 2, leve; 3 a 7, moderada; 8 a 10, intensa. No nosso estudo, as categorias moderada e intensa foram as mais diagnosticadas, demonstrando que os pacientes do apresentavam um elevado nível de dor, como mostra a Tabela 1.

Tabela 1. Nível da sintomatologia dos pacientes com DTM

\begin{tabular}{l|l|l|l|l|l|l}
\hline Variável & \multicolumn{2}{|c|}{ Articular } & \multicolumn{2}{c|}{ Muscular } & \multicolumn{2}{c}{ Total } \\
\hline Sintomatologia & $\mathrm{n}$ & $\%$ & $\mathrm{n}$ & $\%$ & $\mathrm{n}$ & $\%$ \\
\hline Leve & 5 & 100 & $\mathrm{o}$ & $\mathrm{O}, \mathrm{O}$ & 10 & $\mathbf{1 0 0 , 0}$ \\
\hline Moderada & 10 & 66,7 & 5 & 33,3 & 10 & $\mathbf{1 0 0 , 0}$ \\
\hline Intensa & 4 & 40,0 & 6 & 60,0 & 10 & $\mathbf{1 0 0 , 0}$ \\
\hline Total & $\mathbf{1 9}$ & $\mathbf{6 3 , 3}$ & $\mathbf{1 1}$ & $\mathbf{3 6 , 7}$ & $\mathbf{3 0}$ & $\mathbf{1 0 0 , 0}$ \\
\hline
\end{tabular}

A partir das informações constatadas na Tabela 1,25 pacientes apresentaram categorias mais elevadas de dor. Frente a isto, tentou-se constatar a relação entre os níveis de dor (leve e moderada) e a DTM (Tabela 2). Apesar de apresentar níveis maiores de dor, os indivíduos deste estudo foram mais acometidos pela categoria moderada, seguida do nível intenso, e tendo o componente articular como prevalente. Percebeu-se que não houve significância estatística ao verificar a relação entre as categorias de sintomatologia e a DTM, expressos na Tabela 2.

Tabela 2. Nível da sintomatologia dos pacientes com DTM

\begin{tabular}{|c|c|c|c|c|c|c|c|c|}
\hline \multirow{2}{*}{$\begin{array}{l}\text { Variável } \\
\text { Sintomatologia }\end{array}$} & \multicolumn{2}{|c|}{ Articular } & \multicolumn{2}{|c|}{ Muscular } & \multirow[b]{2}{*}{ Qui $^{2}$} & \multirow[b]{2}{*}{$\begin{array}{l}\mathrm{p} \\
\text { valor }\end{array}$} & \multirow[b]{2}{*}{$\mathrm{RP}_{\text {naj }}$} & \multirow[b]{2}{*}{$\begin{array}{l}\text { IC } \\
\text { ( } 95 \%)\end{array}$} \\
\hline & $\mathrm{n}$ & $\%$ & $\mathrm{n}$ & $\%$ & & & & \\
\hline Leve & 5 & 100 & o & 0,0 & 0,800 & 0,371 & 1,50 & $1,049^{-}$ \\
\hline Moderada & 10 & 66,7 & 5 & 33,3 & & & o & 2,145 \\
\hline Total & 15 & 75,0 & 5 & 25,0 & & & & \\
\hline
\end{tabular}

Na Tabela 3, é possível notar uma associação significativa estatisticamente entre a DTM e a intensidade de dor dos pacientes. $\mathrm{O}$ grupo de pacientes com DTM de origem muscular apresentou uma sintomatologia mais aguda. Dessa forma, é possível supor que os pacientes que apresentam DTMs de comprometimento articular buscam o atendimento com mais variações entre as sintomatologias leve, moderada e intensa. Porém, se tem a necessidade de mais estudos a respeito dessa temática para comprovação dessa suposição. Em relação à prevalência da disfunção temporomandibular constatou-se que 19 pacientes tinham desenvolvido a DTM de origem articular, sendo 17 do gênero feminino e 2 do masculino; e 11 pacientes foram diagnosticado com DTM de origem muscular, destes, 2 são do gênero masculino e 9 do feminino. No que diz respeito a faixa etária dos pacientes envolvidos encontrou-se uma proporção de quinze (15) indivíduos para o grupo com menos de 36,5 anos e quinze (15) para o grupo com mais de 36,5 anos.

Tabela 3. Relação entre a DTM e sintomatologia leve e intensa

\begin{tabular}{|c|c|c|c|c|c|c|c|c|}
\hline \multirow{2}{*}{$\begin{array}{l}\text { Variável } \\
\text { Sintomatologia }\end{array}$} & \multicolumn{2}{|c|}{ Articular } & \multicolumn{2}{|c|}{ Muscular } & \multirow[b]{2}{*}{ Qui $^{2}$} & \multirow[b]{2}{*}{$\begin{array}{l}\mathrm{p} \\
\text { valor }\end{array}$} & \multirow[b]{2}{*}{$\mathrm{RP}_{\text {naj }}$} & \multirow[b]{2}{*}{$\begin{array}{l}\text { IC } \\
(95 \%)\end{array}$} \\
\hline & $\mathrm{n}$ & $\%$ & $\mathrm{n}$ & $\%$ & & & & \\
\hline Leve & 5 & 100 & o & 0,0 & 2,813 & 0,044 & 2,50 & $1,170^{-}$ \\
\hline Intensa & 4 & 40,0 & 6 & 60,0 & & & 0 & 5,341 \\
\hline Total & 15 & 75,0 & 5 & 25,0 & & & & \\
\hline
\end{tabular}

DISCUSSÃO

De acordo com o presente estudo determinou-se que não houve associação estatisticamente significante entre as variáveis avaliadas com a DTM, corroborando com os achados de Slade GD et al. ${ }^{8}$, que por meio de estudo de coorte buscou-se definir a etiologia da Disfunção Temporomandibular (DTM) e a sintomatologia dolorosa. Obteve-se numerosos fatores que poderiam implicar na disfunção temporomandibular, como, sexo, idade, estresse, depressão, tratamento ortodôntico, disfunção mastigatória, extração de terceiros molares, traumas faciais e artrite. Com isso ocorre o aparecimento da dor, questionando-se a sua associação com o surgimento e perpetuação da $\mathrm{DTM}^{8}$. Houve uma relação significativa entre a DTM e a intensidade de dor dos pacientes, em que o grupo de pacientes com DTM de origem muscular apresentou uma sintomatologia mais aguda, como também foi encontrado no estudo de Rauhala K et. al., apontando que a dor orofacial associada à Disfunção Temporomandibular tem como principal fator a origem miogênica, sendo que esse tipo de sintomatologia o mais favorável para regredir diante os tratamentos conservadores e mais tradicionais da DTM $^{9,10}$.

Constatou-se que as categorias moderada e intensa foram as mais prevalentes, demonstrando que os pacientes apresentavam um elevado nível de dor indo de acordo com o estudo de Slade GD et al. ${ }^{8}$, no qual foi mostrado que a sintomatologia dolorosa é uma consequência da DTM e não um fator predisponente desta em que indivíduos com DTM crônica possuíam forte relação com dor por pressão nos músculos do trapézio e temporais, possuindo resultados significantes. Os resultados da dor como predisponente para DTM foram escassos $\mathrm{e}$ as estimativas foram consideradas fracas. Ainda nesse estudo, pode-se destacar que pacientes que possuíam DTM transitórias tiveram remissão dos sintomas, ou seja, a dor na DTM sofreu variações de acordo com a 
perpetuação ou não da disfunção temporomandibular, sendo mais um indicativo que a DTM tem potencial causador da dor nessa patologia, acreditando-se que a DTM, quando causa dor, gera um aumento da percepção sensorial dos neurônios nociceptores ${ }^{8}$.

Neste estudo, o componente articular foi o mais prevalente quando relacionado à dor. Estudo conduzido por Freitas LS et al. ${ }^{11}$ demonstrou que patogenicidade da disfunção temporomandibular, se dá, basicamente, pela deterioração da articulação temporomandibular, pelo atrito e abrasão das superfícies articulares presentes nessa estrutura. Essas alterações geram, consequentemente, injúrias teciduais, sendo estas injúrias, um fator precedente para a ativação da cascata de inflamação e do sistema complemento $^{12}$. Assim, promove liberação dos mediadores da inflamação como: Serotonina (5HT) $)^{11}$, as interleucinas IL-4 e IL-6, Prostaglandinas (PG), glutamato, estradiol, receptor Fc III (FCRIII), proteínas Wnt (Wnt-5A), proteína S100A12 (Calgranulina C), as Metaloproteinases (MMPs) e o TNF- $\alpha^{13}$. Além disso, há um aumento de íons Cálcio $\left(\mathrm{Ca}^{2+}\right)$ nas sinapses nervosas, principalmente nos terminais pós-sinápticos dos neurônios, excitam as fibras nervosas tipo $\mathrm{C}$, causando uma sensibilização central, o que resulta na hiperalgesia e aumento dos espasmos musculares, o que contribui como importante fator de fadiga muscular e lesões musculares, consequentemente, a ocorrência da sintomatologia dolorosa a partir da excitação dos neurônios nociceptores na região ${ }^{12,14}$

Assim, a dor atua muito mais como moduladora, agente perpetuador e consequência da disfunção temporomandibular do que como causadora desta patologia, pois para se ter a sintomatologia dolorosa, parte-se do pressuposto de que é preciso ter uma injúria tecidual e, por conseguinte, há a inflamação e o desfecho doloroso, corroborando com os resultados encontrados na tabela 1, pois a percepção da dor se faz mais presente em indivíduos que relataram dor moderada ou intensa. Com isso, estes indivíduos possuem uma chance maior de manutenção positiva da Disfunção Temporomandibular. Tal fato mostra $\mathrm{o}$ caráter modulador e perpetuador da dor na DTM ${ }^{15}$.

$\mathrm{O}$ presente estudo constatou que o sexo feminino é o mais acometido e a maior parte do grupo apresentou idade superior a 36,5 anos, corroborando com Portinho et al. ${ }^{16}$ em estudo de uma série retrospectiva de casos, atendidos entre 2009 e 2012 no ambulatório de cirurgia craniomaxilofacial, com dor ou outros sintomas na região da ATM. Sendo incluídos pacientes acima de 16 anos, independentemente de outras características. Nesse estudo foram avaliados 65 pacientes, sendo $84,6 \%$ do sexo feminino e a idade média dos pacientes foi de $39,9 \pm 14,5$ anos. Os mesmos já tinham sintomas há $11,7 \pm 18$ meses e o quadro era bilateral em $43,1 \%$ dos casos. A dor foi a queixa mais comum, presente em $98,5 \%$ dos pacientes e os demais achados iniciais foram: crepitação ou estalido (56,9\%), desvio lateral ao movimento $(35,4 \%)$, má oclusão $(35,4 \%)$, contratura muscular $(29,2 \%)$, cervicalgia $(27,2 \%)$ e bruxismo $(24,6 \%)$. Havia história de luxação em $6,2 \%$ dos casos e de trauma prévio em $7,7 \%{ }^{16}$.

Este estudo possui limitações, pois como se trata de um estudo transversal, não há como estabelecer relações de causa e efeito. Contudo, instrumentos validados foram utilizados para diminuir a possibilidade de viéses de pesquisa. Estudos longitudinais precisam ser realizados para determinar maior evidência das associações apresentadas neste estudo.

\section{CONCLUSÃO}

Diante dos resultados, conclui-se que o sexo feminino foi o mais acometido pela DTM, possuindo a amostra deste estudo uma idade variando entre $18 \mathrm{e}$ 50 anos. Quanto à origem da disfunção temporomandibular, ocorreu um maior acometimento articular que muscular, e entre os níveis de sintomatologia, moderado e intenso foram os mais diagnosticados. Foi possível determinar que não houve associação estatisticamente significativa entre as variáveis gênero, idade, origem, nível de dor com a DTM. Uma limitação deste trabalho diz respeito ao tipo de estudo que foi realizado, onde a pesquisa foi baseada em um estudo transversal, sendo, portanto, tudo observado e mensurado em uma única vez, sem acompanhamentos futuros.

\section{REFERÊNCIAS}

1. Dworkin SF, LeResche L. Research diagnostic criteria for temporomandibular disorders: review, criteria, examinations and specifications, critique. J Craniomandib Disord. 1992;6(4):301-55.

2. Gonçalves DAG, Bigal ME, Jales LCF, Camparis $\mathrm{CM}$, Speciali JG. Headache and symptoms of temporomandibular disorders: an epidemiologic study. Headache. 2010;50(2):231-41.

3. Piccin CF, Pozzebon D, Chiodelli L, Boufleus J, Pasinato F, Corrêa ECR. Aspectos clínicos e psicossociais avaliados por critérios de diagnóstico para disfunção temporomandibular. Revista Cefac. 2016;18(1):113-19.

4. Winocur E, Emodi-Perlman A. Occlusion, orthodontic treatment and temporomandibular disorders: myths and scientific evidences. in: Orthodonthics-basis aspects and clinical considerations. In Tech. 2012.

5. Jorge JMS, Dini C, Santos L, Camara de Bem SH, Custodio W. Associação entre dimensão vertical de oclusão e transtornos temporomandibulares. ClipeOdonto - UNITAU. 2016;8(1):44- 50.

6. Bayma PTC, Feltrin PP, Dias CAS, Costa JF, Laganá DC, Inoue RT. Temporomandibular disorders in otolaryngology patients. RGO (Porto Alegre). 2010;58(3):313-17. 
7. Martinez JE, Grassi DC, Marques LG. Análise da aplicabilidade de três instrumentos de avaliação de dor em distintas unidades de atendimento: ambulatório, enfermaria e urgência. Rev Bras Reumatol. 2011;51(4):299-308.

8. Slade GD, Ohrbach R, Greenspan JD, Fillingim RB, Bair E, Sanders AE et al. Painful temporomandibular disorder: decade of discovery from OPPERA studies. J Dental Res. 2016; 95(10):1084-92.

9. Rauhala K., Oikarinen KS, Raustia AM. Role of temporomandibular disorders (TMD) in facial pain: occlusion, muscle and TMJ pain. Cranio. 1999;17(4):254-61.

10. Manfredini D, Favero L, Gregorini G, Cocilovo F, Guarda-Nardini L. Natural course of temporomandibular disorders with low painrelated impairment: a 2-to-3-year follow-up study. J Oral Rehabil. 2013;40(6):436-42.

11. Freitas LS. Associação da disfunção Temporomandibular com o polimorfismo 102T-C do gene receptor da serotonina HTR2A [tese]. São José do Rio Preto: Faculdade de Medicina do São José do Rio Preto - FAMERP; 2011.

12. Tanaka E, Detamore MS, Mercuri LG. Degenerative disorders of the temporomandibular joint: etiology, diagnosis, and treatment. J Dent Res. 2008;87(4):296-307.

13. Lopes PRR, Campos PSF, Nascimento RJM. Dor e inflamação nas disfunções temporomandibulares: revisão de literatura dos últimos quatro anos. $\mathrm{R} \mathrm{Ci}$ med biol. 2011; 10(3):317-25.

14. Wang X, Guo H, Wang Y, Yi X. The effects of estrogen on cytoplasmic ca2+ concentration of masticatory muscles myoblast in acid condition. 5th International Conference on Bioinformatics and Biomedical Engineering (iCBBE); 10-12 May 2011; Wuhan, China. Anais. Disponível em http://www.icbbe.org/2011/Proceeding2010.aspx.

15. Cairns BE. Pathophysiology of TMD Pain-Basic Mechanisms and Their Implications for pharmacotherapy. J Oral Rehabil. 2010; 37(6):391-410.

16. Portinho CP, Razera MV, Splitt BI, Gorgen ARH, Faller GJ, Collares MVM. Apresentação clínica inicial em pacientes com disfunção Temporomandibular. Rev Bras Cir Craniomaxilofac. 2012;15(3):109-12.

\section{CONFLITO DE INTERESSES}

Os autores declaram não haver conflitos de interesse.

\section{AUTOR PARA CORRESPONDÊNCIA}

\section{Wyllka Cyntya Galvão da Silva}

wyllka.g@live.com 